\title{
The Comparative Effects of Ankle Bracing on Functional Performance
}

\author{
Thomas M. Newman, Giampietro L. Vairo, and William E. Buckley
}

\begin{abstract}
Ankle sprains represent a common musculoskeletal injury that clinicians are tasked with preventing and treating. Because of the prevalence of this injury, ankle braces have been designed to prophylactically protect the joint and reduce the incidence of repetitive sprains. Although an abundance of literature exists focusing on the efficacy of braces in preventing ankle sprains in young, healthy, and physically active populations, there is a scarcity of evidence specific to the impact of these apparatuses on functional performance; therefore, the purpose of this critically appraised topic (CAT) is to investigate the effects of ankle braces on functional performance measures in such individuals. The outcomes of this CAT will assist sport rehabilitation specialists with informed clinical decision making in managing young, healthy, and physically active populations using ankle braces. Do ankle braces hinder functional performance measures when compared with an unbraced condition in a young, healthy, and physically active population? A minimum of level II evidence research studies were surveyed for this CAT. For this CAT, 1 randomized controlled trial and 3 prospective cohort studies were selected. One study found a statistically significant main effect of increased agility run times while participants wore ankle braces. Another study demonstrated a statistically significant decrease in vertical jump height and ankle range of motion while wearing braces. No other statistically significant findings were reported among studies comparing unbraced with braced conditions. Current data indicate that young, healthy, and physically active individuals may experience varied performance effects when executing specific functional performance tasks while wearing ankle braces. In general, bracing does not appear to significantly impair performance on most functional tasks; however, decrements were noted to increases in agility run time and decreases in vertical jump height. Subsequent analysis indicated that a brace may result in decreased ankle plantarflexion, dorsiflexion, eversion, and inversion range of motion, which may underpin noted performance deficits.
\end{abstract}

Keywords: brace, sprain, sport, outcomes, evidence-based practice

\section{Clinical Scenario}

Ankle sprains represent a common musculoskeletal injury encountered in physical activity that clinicians are tasked with preventing and treating. Because of the prevalence of this injury, various ankle braces have been designed to prophylactically protect the joint and reduce the incidence of repetitive sprains. Although an abundance of literature exists focusing on the efficacy of braces in preventing ankle sprains in young, healthy, and physically active populations, there is a scarcity of evidence specific to the impact of these apparatuses on functional performance; therefore, the purpose of this critically appraised topic (CAT) is to investigate the effects of ankle braces on functional performance measures in such individuals. The outcomes of this CAT will assist sport rehabilitation specialists with informed clinical decision making in managing young, healthy, and physically active populations using ankle braces.

\section{Focused Clinical Question}

Do ankle braces hinder functional performance measures when compared with an unbraced condition in a young, healthy, and physically active population?

\footnotetext{
The authors are with the Department of Kinesiology, The Pennsylvania State University, University Park, PA. Vairo is also with the Department of Orthopaedics and Rehabilitation, University Park Regional Campus, PA. Newman (tmn5003@ gmail.com) is corresponding author.
}

\section{Summary of Search, "Best Evidence" Appraised, and Key Findings}

- A minimum of level II evidence research studies were surveyed for this CAT. ${ }^{1,2}$

- For this CAT, 1 randomized controlled trial ${ }^{3}$ and 3 prospective cohort studies were selected. ${ }^{4-6}$

- One study found a statistically significant main effect of increased agility run times while participants wore ankle braces. ${ }^{4}$

- Another study demonstrated a statistically significant decrease in vertical jump height. ${ }^{6}$

- No other statistically significant findings were reported among studies comparing unbraced with braced conditions. ${ }^{3-6}$

O The following functional performance measures were used in this CAT: Sargent Chalk Jump Test, Vertec vertical jump, soccer ball kicking accuracy, right-boomerang run test, southeast Missouri configuration, 40 -yd dash, $\mathrm{S} 180^{\circ}$ agility test, and $t$ test. ${ }^{3,4,6}$

O Brace conditions included in this study were unbraced, laceup, semirigid, and Seattle Ankle Orthosis braces.

- A statistically significant decrease in ankle range of motion (ROM) was reported while wearing braces. ${ }^{6}$

O Plantarflexion, dorsiflexion, eversion, and inversion

\section{Clinical Bottom Line}

Current data indicate that young, healthy, and physically active individuals may experience varied performance effects when 
executing specific functional performance tasks (see the "Appendices" section) while wearing ankle braces. In general, bracing does not appear to cause statistically significant impairments to performance on most functional tasks; however, decrements were noted to yield increases in agility run time and decreases in vertical jump height, which may create real-world deficits. A subsequent effect size analysis indicated that a brace may result in decreased ankle plantarflexion, dorsiflexion, eversion, and inversion ROM, which may underpin noted performance deficits.

\section{Strength of Recommendation}

A grade of $\mathrm{B}^{7}$ or moderate level of evidence exists demonstrating that ankle braces may have varied impacts on functional performance measures. We base this statement from the literature's reported statistical significance outcomes. To supplement this assessment, we also offer an independent post hoc analysis of related measures, which may provide an evaluation lending to clinical significance to augment our current strength of recommendation.

\section{Search Strategy}

\section{Terms Used to Guide Search Strategy}

- Patient/Client group: Young, healthy, and physically active individuals

- Intervention (or assessment): Ankle braces

- Comparison: Unbraced or no ankle brace

- Outcomes: Functional performance measures

\section{Sources of Evidence Search}

- PubMed

- The Cochrane Library

- CINAHL

- PEDro

- SPORTDiscus

\section{Inclusion and Exclusion Criteria}

\section{Inclusion Criteria}

- Original research studies evaluating the use of ankle braces during functional performance

- Limited to English language

- Limited to human participants

- Limited to the previous 15 years (2002-2016)

- A minimum of level II evidence

\section{Exclusion Criteria}

- Research studies that did not compare a braced condition with an unbraced condition

- Research studies that did not assess functional performance measures

- Research studies reporting duplicate outcomes data

- Pilot or case studies

- Animal model experiments

\section{Results of Search}

Thirty-two articles were identified incorporating the related keywords in the defined electronic databases. Most publications did not incorporate an ankle brace intervention or did not utilize functional performance measures; furthermore, some studies did not incorporate a control condition or sampled an injured population. Of these, only 4 articles were selected to be included in this CAT (Figure 1).

The included articles were classified according to the Centre for Evidence-Based Medicine (Oxford, UK). ${ }^{1}$ Results of the literature search are listed in Table 1. A PEDro score was used to scrutinize the lone randomized controlled trial. ${ }^{8}$ To bolster the qualitative appraisal of the included works, the Downs and Black revised checklist was used for assessing methodological quality for all articles employed in this CAT (Table 2). ${ }^{9}$

\section{Best Evidence}

Table 3 identifies the best available evidence based on the literature review. All studies were ranked at a minimum of level II evidence and incorporated the use of an ankle brace compared with an unbraced condition during various functional performance measures. Comparison with other bracing conditions was also included in this review. Post hoc analyses were conducted using effect sizes and were categorized according to Cohen. ${ }^{10}$

\section{Implications for Practice, Education, and Future Research}

Ankle injuries during physical activity are the most common type of musculoskeletal injury during activity, with up to $85 \%$ of these injuries classified as ankle sprains. ${ }^{4}$ To reduce the incidence of sprains, especially in those with a history of ankle injury, ${ }^{3}$ the use of prophylactic ankle braces in young, healthy, and physically active populations is common. Despite empirical data suggesting ankle bracing as an efficacious injury prevention intervention, the values of patients, clients, sport rehabilitation specialists, and related administrative personnel may influence the decision to employ or refute the use of such apparatuses due to concern for hindering functional performance capacity. Although anecdotal, such concerns should be considered from the perspective of the sport rehabilitation specialist as a means to address the multifaceted components encountered with clinical decision making given the impact braces may have on athletic capacity, or task-specific aptitude with an individual's participation in sports, or technical job-related demands, respectively.

Based on the results of our literature survey, inferences from statistically significant findings suggest that ankle bracing may only yield specific impairments with the right-boomerang run test ${ }^{4}$ and vertical jump height using the Vertec system ${ }^{6}$ when compared with an unbraced control condition; therefore, it appears that ankle bracing does not appear to significantly impede most functional performance tasks as determined from various balance, ${ }^{4-6}$ sprint, ${ }^{3}$ agility,,$^{3,4,6}$ and sport-specific ${ }^{3}$ activities. Both Ambegaonkar et $\mathrm{al}^{4}$ and Parsley et $\mathrm{al}^{6}$ suggested that their noted performance deficits may have been attributed to potential decreases in joint ROM while wearing the ankle apparatuses. Ankle braces have been shown to decrease talocrural and subtalar joint ROM by mean differences of $14.9^{\circ}$ for inversion, $14.4^{\circ}$ for eversion, $3.2^{\circ}$ for dorsiflexion, and $9.3^{\circ}$ for plantarflexion in a lace-up braced condition when compared with an unbraced condition. ${ }^{11}$ Similarly, a semirigid braced 


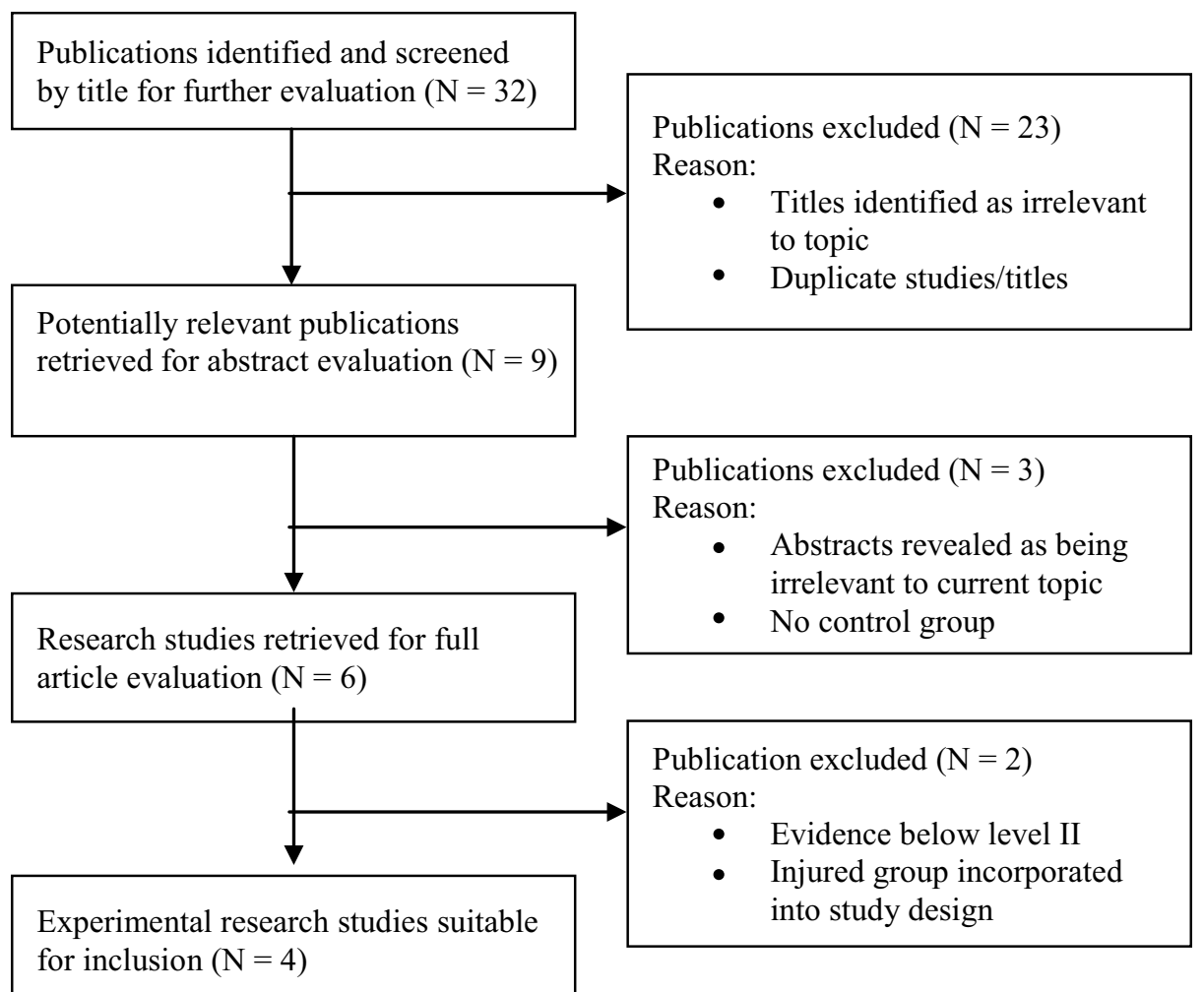

Figure 1 - Quorom statement flow diagram illustrating the results of the literature search.

condition has been found to further restrict ankle ROM by mean differences of $20.2^{\circ}$ for inversion and $19.8^{\circ}$ for eversion when compared with an unbraced condition. ${ }^{11}$ In an effort to investigate whether a similar trend existed in the current appraised articles of this CAT, we used the data of Parsely et $\mathrm{al}^{6}$ to conduct a post hoc analysis of ankle ROM when comparing unbraced and braced conditions (Figure 2). Based on our post hoc findings, ankle bracing produces large magnitude decreases in plantarflexion with a lace-up apparatus and large magnitude decreases in inversion when wearing a Seattle Ankle Orthosis (Figure 2). Although ankle braces are designed to limit extreme ROM, particularly in the frontal plane, for preventing injury, it may be that concomitant functional performance deficits may arise from these joint excursion decrements.

As a result of decreases in joint ROM, impairments to agility run time and vertical jump height may be associated with braces hindering the stretch-shortening cycle at the ankle. This cycle is characterized by a constant transfer from the preactivated and

Table 1 Summary of Experimental Designs for Articles Retrieved

\begin{tabular}{|c|c|c|c|}
\hline Level of evidence & Experimental design & Number located & Reference \\
\hline I & Randomized controlled trial & 1 & Putnam et $\mathrm{al}^{3}$ \\
\hline II & Prospective cohort & 3 & $\begin{array}{l}\text { Ambegaonkar et } \mathrm{al}^{4} \\
\text { Hardy et } \mathrm{al}^{5} \\
\text { Parsley et } \mathrm{al}^{6}\end{array}$ \\
\hline
\end{tabular}

Table 2 Downs and Black's Revised Checklist for Measuring Study Quality (Scores by Article)

\begin{tabular}{lcccccccccccccccccccccccccccccc}
\hline Article & $\mathbf{1}$ & $\mathbf{2}$ & $\mathbf{3}$ & $\mathbf{4}$ & $\mathbf{5}^{\mathbf{a}}$ & $\mathbf{6}$ & $\mathbf{7}$ & $\mathbf{8}$ & $\mathbf{9}$ & $\mathbf{1 0}$ & $\mathbf{1 1}$ & $\mathbf{1 2}$ & $\mathbf{1 3}$ & $\mathbf{1 4}$ & $\mathbf{1 5}$ & $\mathbf{1 6}$ & $\mathbf{1 7}$ & $\mathbf{1 8}$ & $\mathbf{1 9}$ & $\mathbf{2 0}$ & $\mathbf{2 1}$ & $\mathbf{2 2}$ & $\mathbf{2 3}$ & $\mathbf{2 4}$ & $\mathbf{2 5}$ & $\mathbf{2 6}$ & $\mathbf{2 7}$ & $\mathbf{T o t a l}^{\mathbf{b}}$ \\
\hline $\begin{array}{l}\text { Ambegaonkar } \\
\text { et al }\end{array}$ & 1 & 1 & 1 & 1 & 0 & 1 & 1 & 0 & 0 & 1 & 0 & 0 & 1 & 0 & 0 & 1 & 1 & 1 & 1 & 1 & 1 & 0 & 1 & 0 & 0 & 0 & 0 & 15 \\
${\text { Hardy et } \mathrm{al}^{5}}$ & 1 & 1 & 1 & 1 & 0 & 1 & 1 & 0 & 0 & 0 & 1 & 0 & 1 & 0 & 0 & 1 & 1 & 1 & 1 & 1 & 1 & 0 & 1 & 0 & 0 & 0 & 0 & 15 \\
${\text { Parsley et } \mathrm{al}^{6}}$ & 1 & 1 & 1 & 1 & 0 & 1 & 1 & 0 & 0 & 1 & 0 & 0 & 0 & 0 & 0 & 0 & 1 & 1 & 1 & 1 & 1 & 0 & 1 & 0 & 0 & 0 & 0 & 13 \\
Putnam el al$^{3}$ & 1 & 1 & 1 & 1 & 0 & 1 & 1 & 0 & 0 & 1 & 1 & 0 & 1 & 0 & 0 & 0 & 0 & 1 & 1 & 1 & 1 & 0 & 1 & 0 & 0 & 0 & 1 & 15 \\
\hline
\end{tabular}

Note: $1=$ yes; $0=$ no or unable to be determined based on available information.

${ }^{\mathrm{a}} 2=$ yes; $1=$ partially; $0=$ no. ${ }^{\mathrm{b}} 5$-point scale; $0=$ no. 


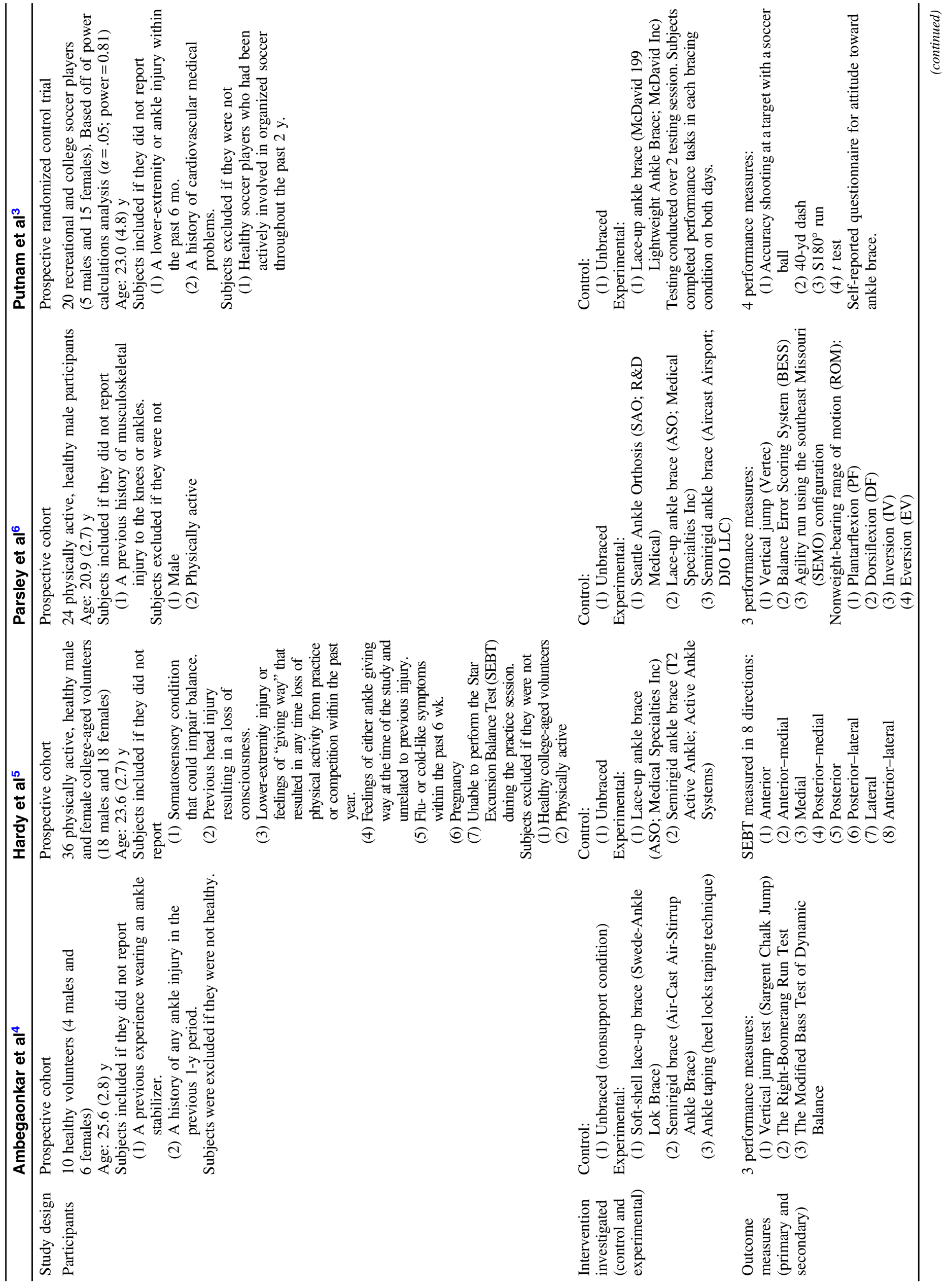




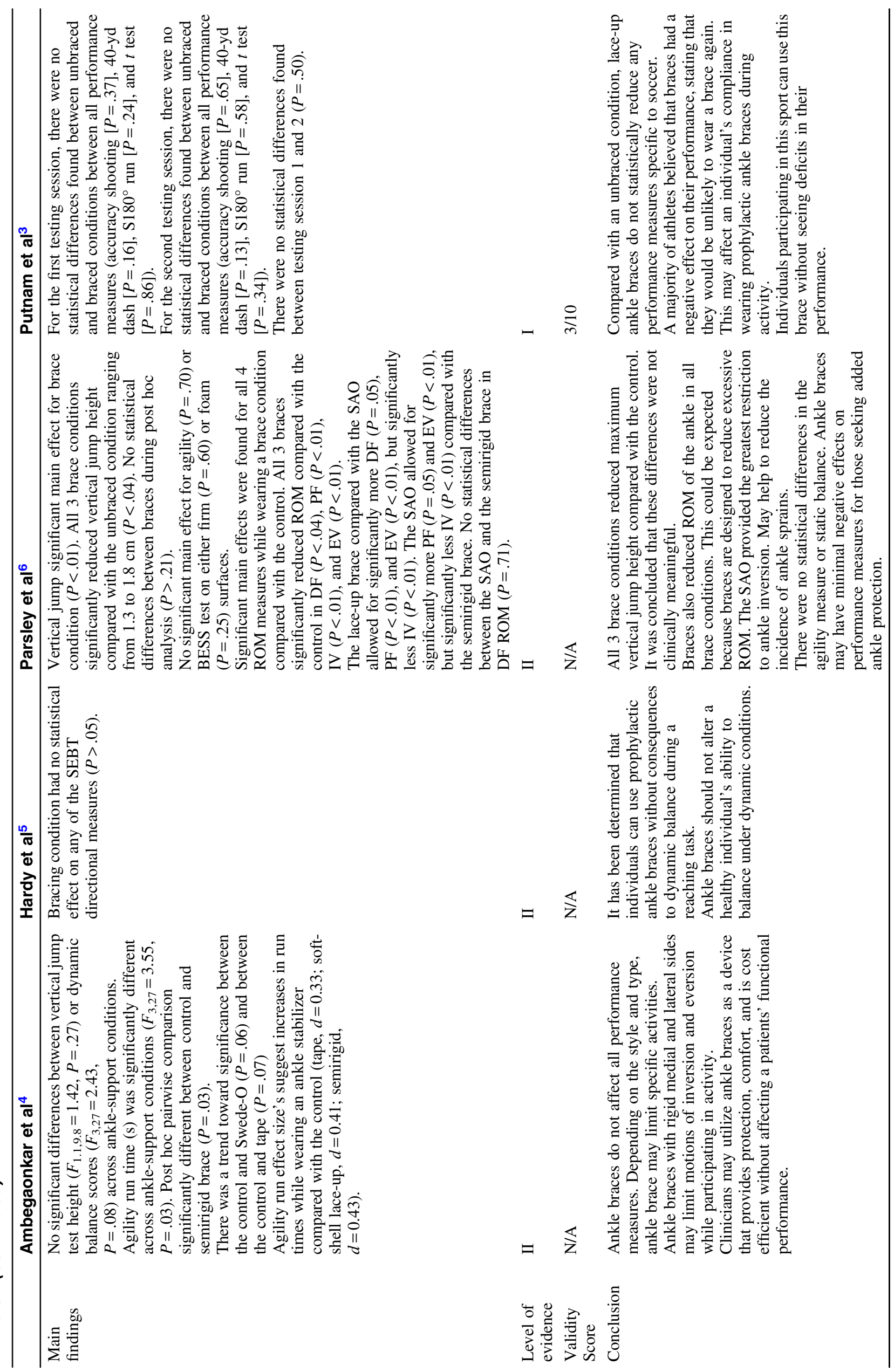




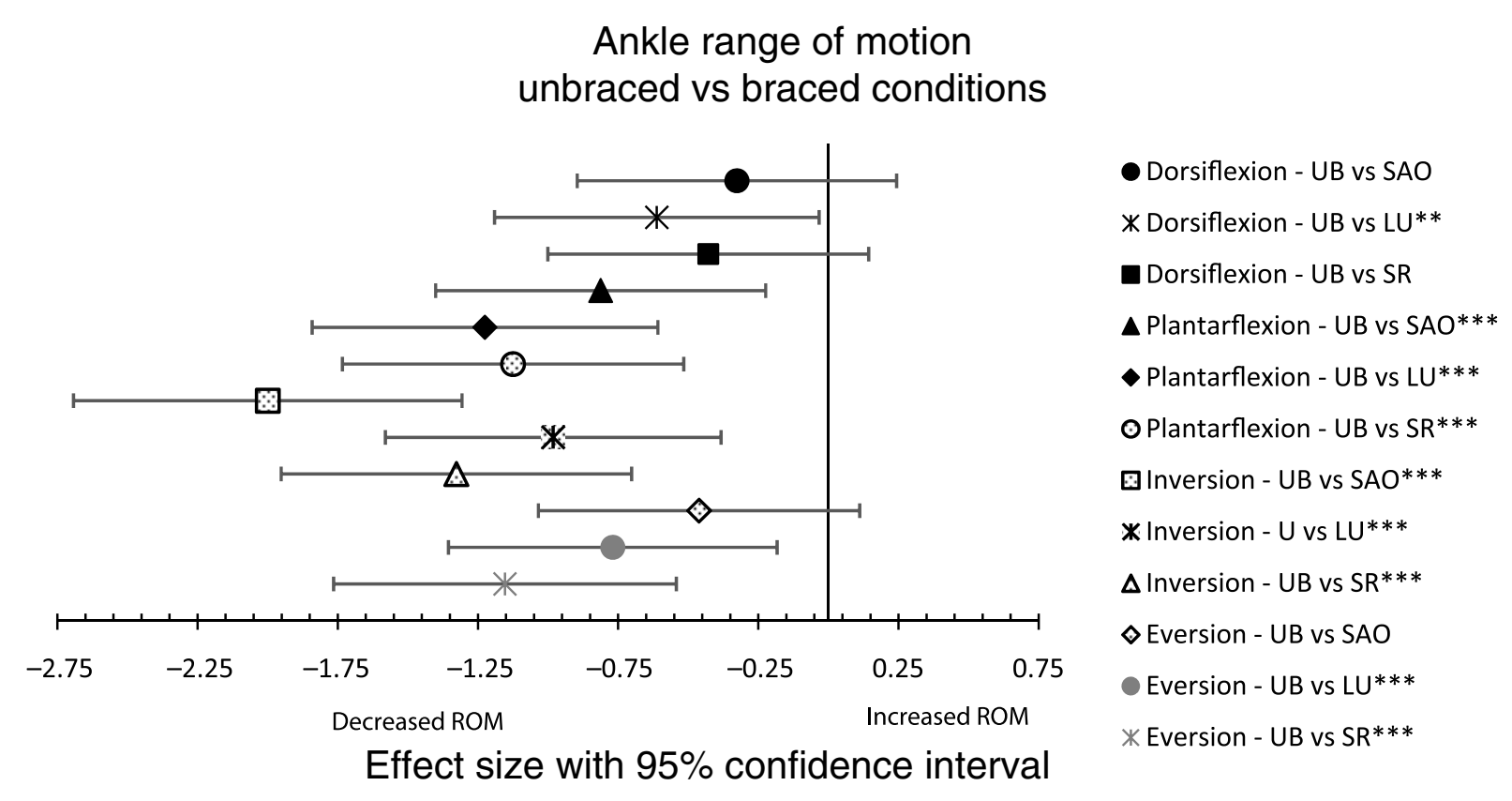

Figure 2 - Post hoc ankle range of motion analysis. LU indicates lace-up; ROM, range of motion; SAO, Seattle Ankle Orthosis; SR, semirigid; $\mathrm{UB}$, unbraced. $* *$ Medium effect size $\geq 0.5$ to $<0.8 .{ }^{10} * * *$ Large effect size $\geq 0.8 .{ }^{10}$

eccentrically lengthened muscle-tendon complex to the concentric push off, which is utilized in most activities such as running or hoping. ${ }^{12}$ As the muscle lengthens, elastic energy is stored until it is used to help generate increased force during the shortening or push off phase of locomotion. ${ }^{12}$ Bracing the ankle, which restricts ROM in the sagittal and frontal planes, may impede the ability of the triceps surae musculotendinous unit and other lower-extremity musculature to generate forces necessary for peak performance when compared with an unbraced condition.

To aid in clinical decision making, employing not only statistical significance as a means to discern whether ankle braces definitely impair functional performance but also recognizing the magnitude of change represents an additional factor to consider for weighing statistical in conjunction with potential clinical significance. The heterogeneity of measures presented across the literature in this CAT prevented collapsing the data for an abbreviated meta-analysis; however, given the means and standard deviations found among the research studies, as with the ROM measures, we were able to calculate effect sizes and associated $95 \%$ confidence intervals to provide additional insights for drawing informed conclusions from our focused clinical question. It is also important to note that the type of braces used in these included studies differed by style of brace and by manufacturer. When appraising an array of different ankle braces among available studies, it is paramount to appreciate the variance in bracing types (eg, rigid, semirigid, or lace-up) and materials used. These minor design and construction differences may produce greater ROM restrictions compared with another brace, leading to statistically significant differences on functional performance and balance measures while also making it challenging for direct comparisons.

Our post hoc analysis of balance focused on 3 different field tests (Figure 3). These measures were assessed using either reach distances, ${ }^{5}$ an error scoring system that summed total errors, ${ }^{6}$ or subtracting penalties from a max of 100 points. ${ }^{4}$ Our calculated effect sizes trended toward either a neutral or small ${ }^{11}$ negative effect while wearing a brace condition. This held true except for the Modified Bass Test where individuals demonstrated a large positive effect size while wearing a lace-up brace condition, but moderate impairment while wearing a semirigid brace. The Modified Bass Test forces participants to jump to new positions compared with the more stationary Star Excursion Balance Test and Balance Error Scoring System test. Because of the restrictive nature of ankle braces, the lace-up condition may have provided additional stability to subjects while they completed the task, whereas the semirigid ankle brace may have created too much restriction and impeded the ability of participants to complete the task successfully.

The neutral to small effect sizes noted with Star Excursion Balance Test outcomes may be attributed to participants using a compensatory knee or hip joint movement strategy to offset limited ankle ROM in maintaining comparable reach distances when compared with an unbraced condition. With a more static balance test like the Balance Error Scoring System, which requires minimal ankle joint excursion, the influence of a brace on performance in this task may not have been a significant factor. An improvement in the Modified Bass Test with a lace-up brace may be potentially explained by the apparatus augmenting joint stability as well as improving proprioception and neuromuscular control while performing this specific task; however, the contrasting deficit noted with this same task while wearing a semirigid brace may be attributed to an increased limitation in ankle ROM when compared with a lace-up brace. Compared with the Star Excursion Balance Test and Balance Error Scoring System, the Modified Bass Test requires an individual to propel the center of mass in a series of directions similar to a single-leg hop test. Greater ROM limitations placed upon the ankle in a semirigid brace would theoretically result in a greater negative impact on the stretch-shortening cycle that may be interpreted as yielding a moderate performance deficit in this task when compared with a less restrictive lace-up brace.

Effect sizes for sprint and agility run tasks are presented in Figure 4 and show only 2 functional performance measures with 


\section{Dynamic balance unbraced vs braced conditions}

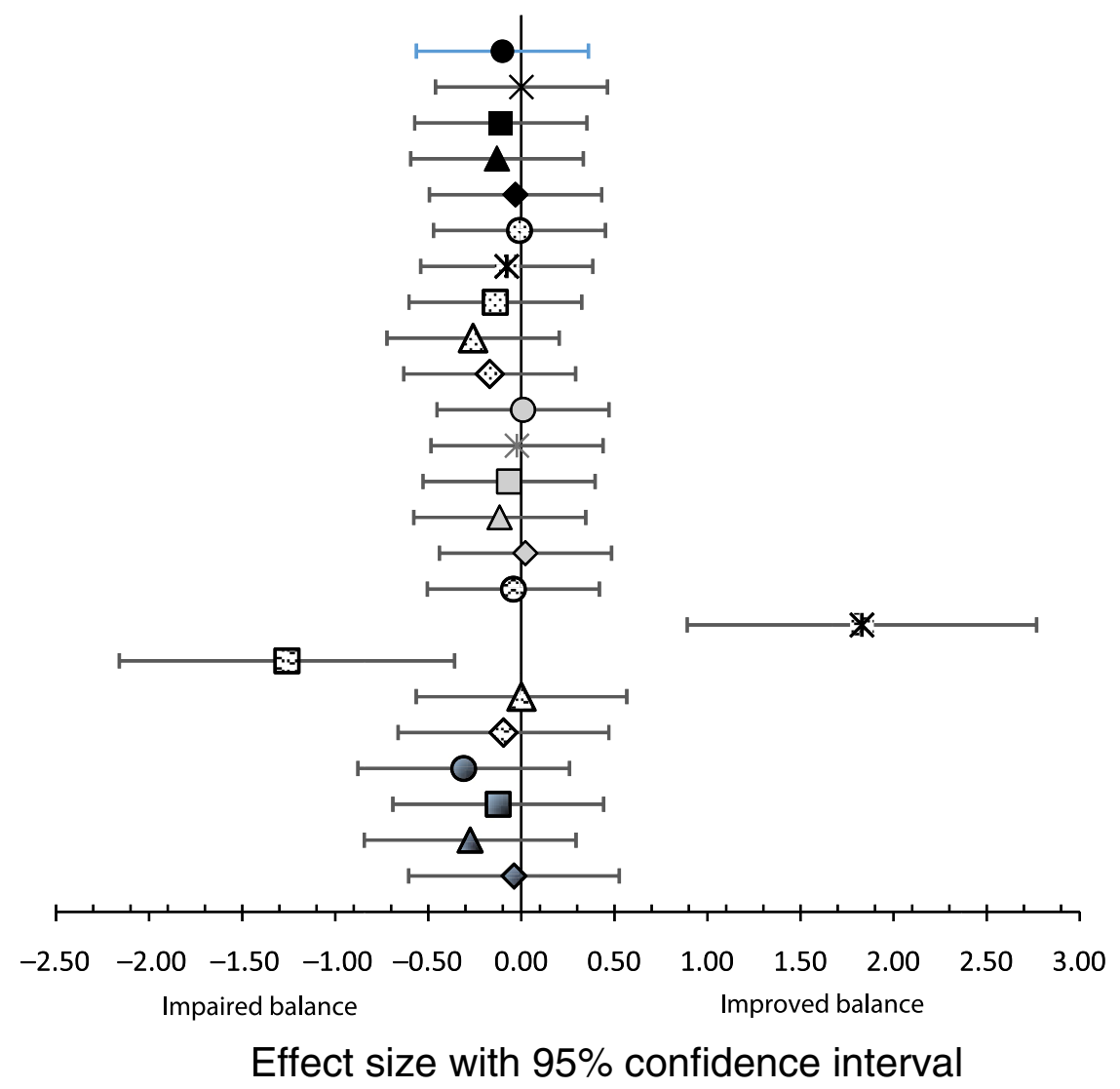

- SEBT anterior - UB vs LU

* SEBT anterior - UB vs SR

- SEBT anterior-medial - UB vs LU

$\triangle$ SEBT anterior-medial - UB vs SR

$\diamond$ SEBT medial - UB vs LU

OSEBT medial - UB vs SR

* SEBT posterior-medial - UB vs LU

$\square$ SEBT posterior-medial - UB vs SR

$\triangle$ SEBT posterior - UB vs LU

$\diamond$ SEBT posterior - UB vs SR

OSEBT posterior-lateral - UB vs LU

* SEBT posterior-lateral - UB vs SR

$\square$ SEBT lateral - UB vs LU

$\triangle$ SEBT lateral - UB vs SR

$\diamond$ SEBT anterior-lateral - UB vs LU

OSEBT anterior-lateral - UB vs SR

* Modified bass - UB vs LU***

Modified bass - UB vs SR**

$\triangle B E S S$ firm - UB vs SAO

$\diamond$ BESS firm - UB vs LU

O BESS firm - UB vS SR

BESS foam - UB vs SAO

$\triangle$ BESS foam - UB vs LU

$\diamond$ BESS foam - UB vs SR

Figure 3 - Post hoc balance analysis. BESS indicates Balance Error Scoring System; LU, lace-up; SAO, Seattle Ankle Orthosis; SEBT, Star Excursion Balance Test; SR, semirigid; UB, unbraced. $* *$ Medium effect size $\geq 0.5$ to $<0.8 .^{10} * * *$ Large effect size $\geq 0.8$. ${ }^{10}$

trends for small, negative effect sizes. Both brace conditions of the right-boomerang run test ${ }^{4}$ increased run times compared with the unbraced condition. Although only one functional performance measure indicates an increase in run times, Figure 4 depicts a negative trend for most measures while wearing ankle braces. By placing ankle braces on the lower limb, it is possible that normal movement patterns are limited by these devices, and in doing so, it limits the ability of the ankle to propel the body with the same amount of force. In addition, ankle braces that are designed with more rigid medial and lateral side supports may limit subtalar motion of the ankle, decreasing the ability for the participant to move quickly from side to side. Although not statistically significant, Putnam et $\mathrm{al}^{3}$ presented data that those wearing ankle braces had an increased 40-yd dash time between 0.11 and 0.12 seconds. During competition, this difference in time may be the reason for winning or losing an event. For those individuals serving in various emergency services and military positions, any deficit in sprint or agility speed may pose unforeseen risks greater than musculoskeletal ankle sprain, such as not being able to move as quickly away from the blast radius of an explosive device. Even though most functional performance measures did not show clinical or statistical significance, specialists working with individuals in a competitive environment should understand that negative effects may arise.
Post hoc analyses of vertical jump height and sport-specific activities are presented in Figure 5. A trend toward a small negative effect was found in all Vertec jump comparisons, as well as in session one soccer ball kicking accuracy in those individuals wearing ankle braces. Limiting ROM and subsequently reducing the ability for the ankle to propel or generate force, an individual may not be able to jump as high or place their lower-extremity in the proper position to make accurate shots. For example, if a football wide receiver is wearing ankle braces and has to make a vertical leap to catch a football, ankle braces may limit the ability for that athlete to jump high enough to catch the ball. Besides, from a clinical standpoint, it is paramount that any devices that are added to an athlete or individual do not place them in a position of greater danger. If the athlete cannot propel themselves out of harm's way due to the restrictive nature of the ankle braces, this may create an injury at another location other than the ankle.

There are limitations associated with this CAT, including variability in the types of functional performance test used, especially as it pertains to measures of vertical jump, agility, and balance. Although this may limit the inferences from this specific body of work, it does appropriately reflect the myriad of different methods a practitioner may employ in real-world clinical or applied settings. Neutral or negative clinically significant trends were 


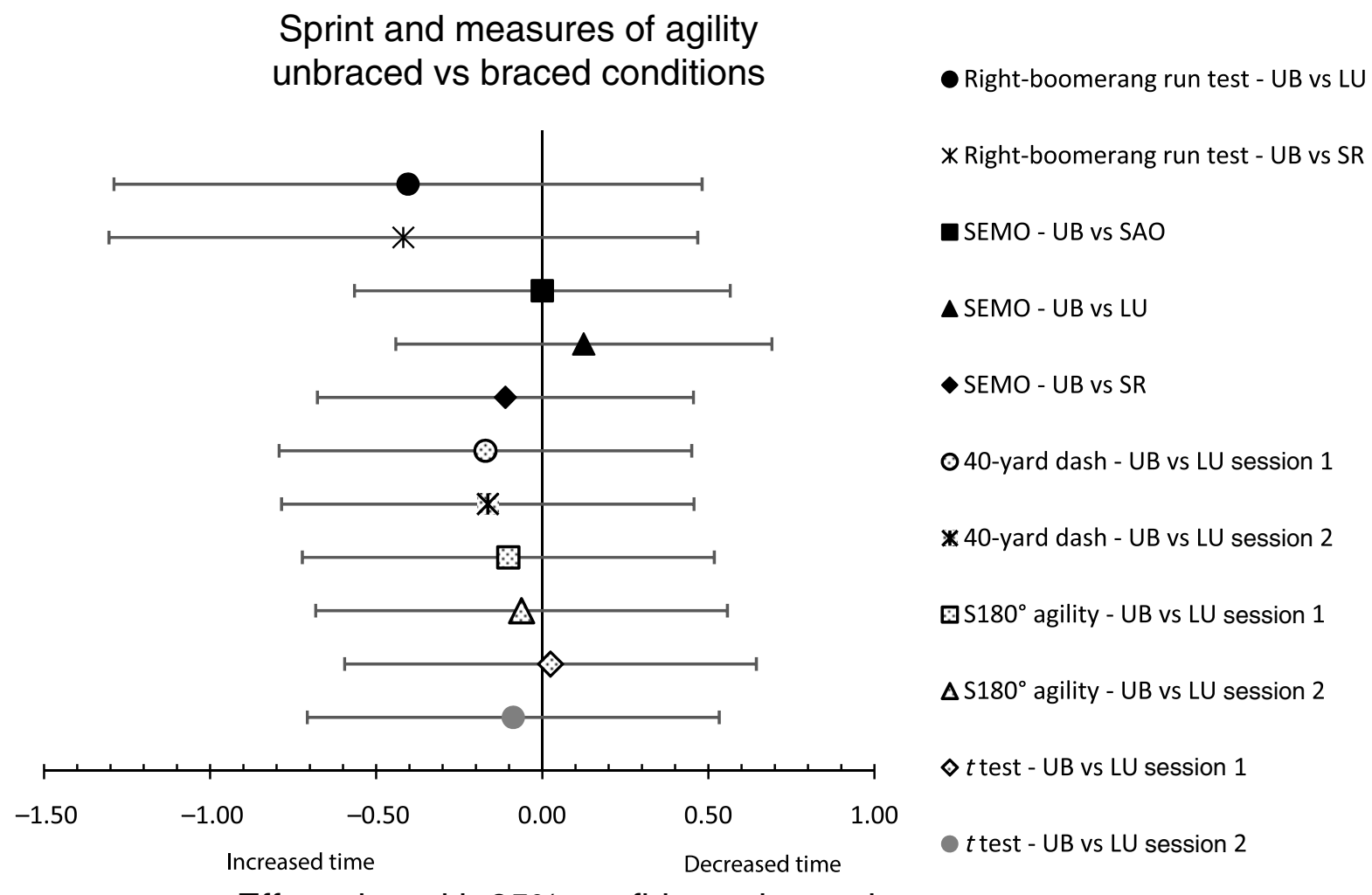

Effect size with 95\% confidence interval

Figure 4 - Post hoc sprint and agility measures analysis. LU indicates lace-up; SAO, Seattle Ankle Orthosis; SEMO, southeast Missouri configuration; SR, semirigid; UB, unbraced.

\section{Vertical jump and sport-specific activity} unbraced vs braced conditions

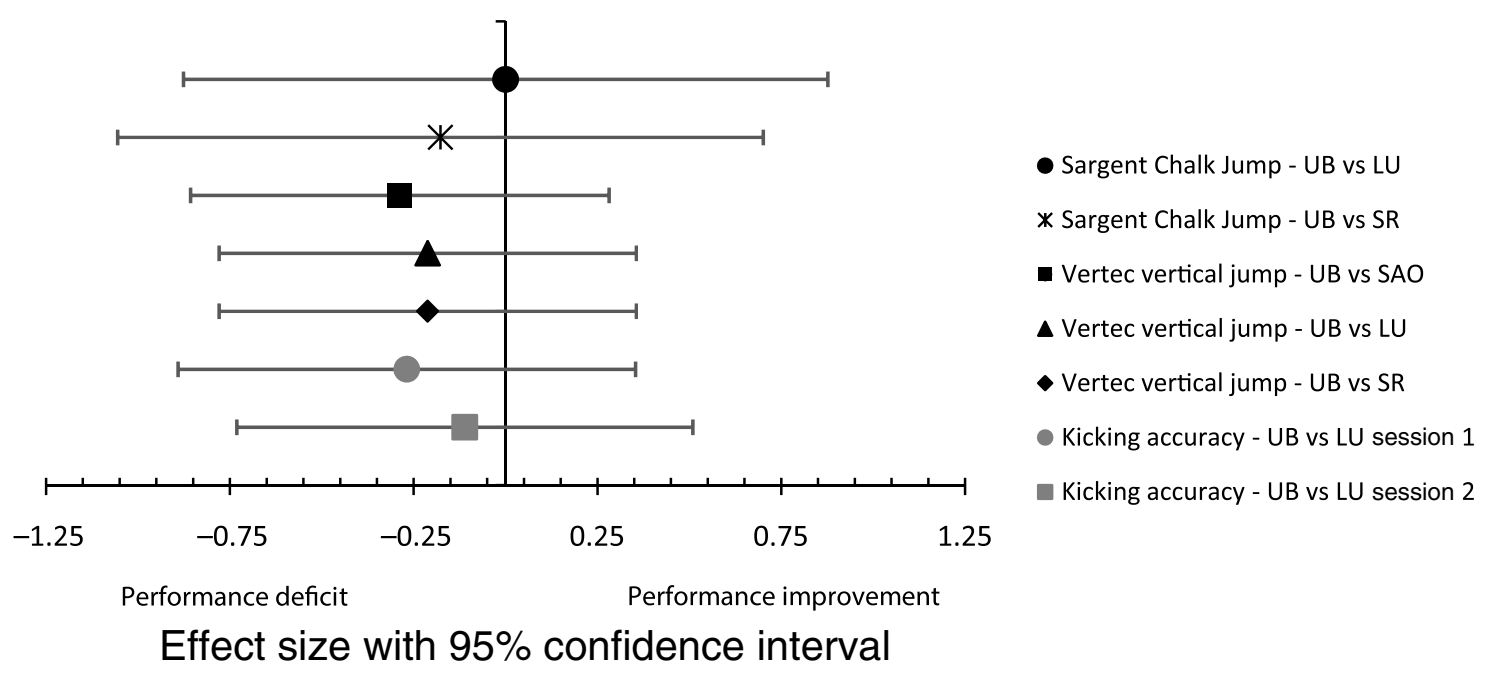

Figure 5 - Post hoc vertical jump height and sport-specific activity analysis. LU indicates lace-up; SAO, Seattle Ankle Orthosis; SR, semirigid; $\mathrm{UB}$, unbraced. 
found using $95 \%$ confidence intervals; unfortunately many crossed the zero line indicating a not statistically significant effect size. Other limitations stem from using articles only published in the English language, and the fact that our outcomes are currently limited to young, healthy, and physically active individuals; therefore, practitioners should use caution in extrapolating our findings to other populations not represented in this CAT. In addition, some articles were eliminated from this study because it was not clearly defined whether a physically active population or sample was being used, thus this may have removed some studies relevant to this CAT. Based on these limitations, further research should be conducted as a means to better clinical decision making when weighing the advantages and disadvantages of using such apparatuses. Nonetheless, the outcomes of this CAT may help sports medicine professionals, athletes, and other individuals who participate in sports or physical activities become more aware of the potential impact ankle braces may have on functional performance.

\section{References}

1. OCEBM Levels of Evidence Working Group. The Oxford 2011 Levels of Evidence. Oxford, UK: Oxford Centre for Evidence-Based Medicine; 2011.

2. McKeon P, Medina J, Hertel J. Hierarchy of evidence-based clinical research in sports medicine. Athletic Ther Today. 2006;11:42-45.

3. Putnam AR, Bandolin SN, Krabak BJ. Impact of ankle bracing on skill performance in recreational soccer players. $P M$ R. 2012;4(8): 574-579. PubMed doi:10.1016/j.pmrj.2012.04.001
4. Ambegaonkar JP, Redmond CJ, Winter C, et al. Ankle stabilizers affect agility but not vertical jump or dynamic balance performance. Foot Ankle Spec. 2011;4(6):354-360. PubMed doi:10.1177/ 1938640011428509

5. Hardy L, Huxel K, Brucker J, Nesser T. Prophylactic ankle braces and star excursion balance measures in healthy volunteers. J Athl Train. 2008;43(4):347-351. PubMed doi:10.4085/1062-6050-43.4.347

6. Parsley A, Chinn L, Lee SY, Ingersoll C, Hertel J. Effect of 3 different ankle braces on functional performance and ankle range of motion. Athl Train Sports Health Care. 2013;5(2):69-75. doi:10.3928/ 19425864-20130213-02

7. Ebell MH, Siwek J, Weiss BD, et al. Strength of recommendation taxonomy (SORT): a patient-centered approach to grading evidence in the medical literature. J Am Board Fam Pract. 2004;17(1):59-67. doi:10.3122/jabfm.17.1.59

8. Maher CG, Sherrington C, Herbert RD, Moseley AM, Elkins M. Reliability of the PEDro scale for rating quality of randomized controlled trials. Phys Ther. 2003;83(8):713-721. PubMed

9. Downs SH, Black N. The feasibility of creating a checklist for the assessment of the methodological quality both of randomised and non-randomised studies of health care interventions. J Epidemiol Community Health. 1998;52(6):377-384. doi:10.1136/jech.52.6.377

10. Cohen J. Statistical Power Analysis for the Behavioral Sciences. 2nd ed. Hillsdale, NJ: L. Erlbaum; 1988.

11. Cordova M, Ingersoll C, LeBlanc M. Influence of ankle support on joint range of motion before and after exercise: a meta-analysis. J Orthop Sports Phys Ther. 2000;30(4):170-177. PubMed doi: 10.2519/jospt.2000.30.4.170

12. Komi PV. Stretch-shortening cycle. In: Komi PV, ed. Strength and Power in Sport. 2nd ed. Blackwell Science Publications; 2002:184-202.

\section{Appendix A: Star Excursion Balance Test ${ }^{5}$}

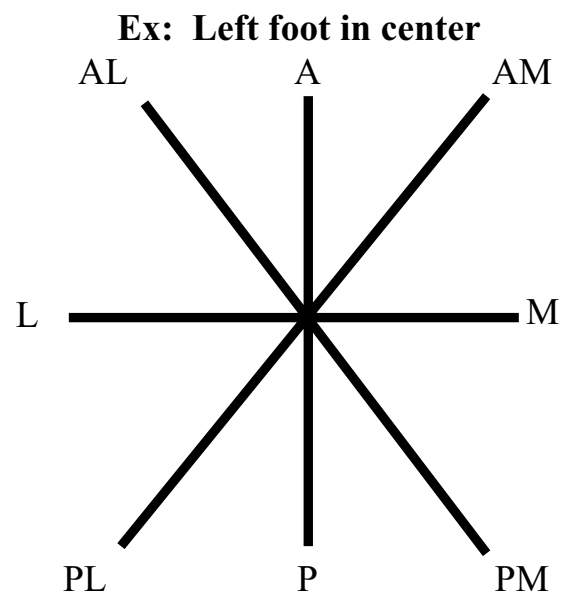

The Star Excursion Balance Test (SEBT) is a multidirectional functional test designed to measure dynamic balance. The SEBT is performed with a subject standing at the center of a grid placed on the floor, with 8 lines radiating out from the center. These lines are separated by $45^{\circ}$ increments and are directionally labeled based on the stance limb. They are as follows: anterolateral (AL); anterior (A); anteromedial (AM); medial (M); posteromedial (PM); posterior (P); posterolateral (PL); and lateral (L). Instructions are given for individuals to reach as far as possible with the nonstance limb in each of the 8 directions. This is generally completed on both limbs for comparison purposes. 


\section{Appendix B: Modified Balance Error Scoring System Test ${ }^{6}$}

The modified Balance Error Scoring System test has individuals stand in one of 3 testing positions with their hands on their iliac crests and eyes closed. These 3 testing positions are as follows: feet together; single limb stance on the nondominant limb; and tandem stance with the nondominant limb placed behind the dominant limb. These tests are performed both on a firm surface and on an Airex Pad, each for 20 seconds. Errors are any of the following: (1) opening eyes; (2) lifting the forefoot of the heel; (3) stepping or stumbling; (4) removing the hands from the iliac crests; (5) moving the hip more than $30^{\circ}$ of flexion or abduction; and (6) being out of position for more than 5 seconds.

\section{Appendix C: The Modified Bass Test for Dynamic Balance ${ }^{4}$}
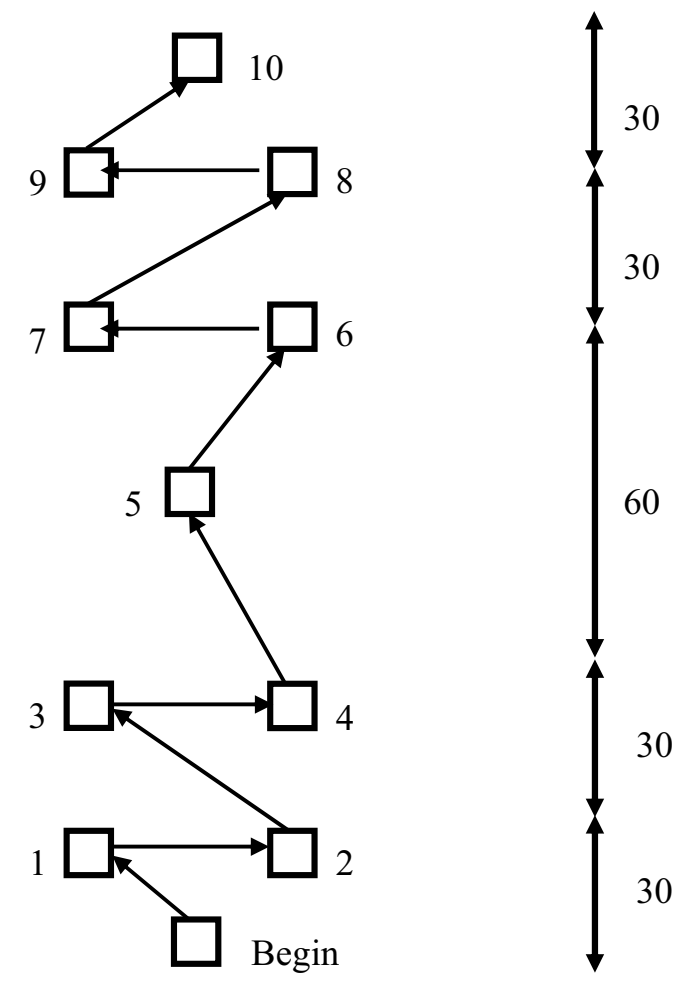

30

Subjects begin in a 1-limb stance with the right foot on the starting mark. They are then instructed to leap to the first mark with the left foot and to hold steady on the ball of the foot for up to 5 seconds. They will then jump to the second marker with the right foot and alternate limbs from marker 1 to 10 . A max of 100 points may be awarded for a perfect test. Five points are removed if a subject misses the intended mark. One point is deducted for each second the subject is not able to balance out of 5 seconds.

\section{Appendix D: Sargent Chalk Jump Test ${ }^{4}$}

Individuals stand with the dominant limb toward the wall with heels together and hold a 2.5-cm piece of chalk in the hand nearest the wall. Keeping heels on the floor, the individual will reach as high as possible and make a baseline mark on the wall. Individuals will then crouch down and jump as high as possible and place a mark at the apex of the jump. The difference between the baseline standing reach and the mark at the apex of the jump is measured in inches as the vertical jump.

\section{Appendix E: Vertec Vertical Leap ${ }^{6}$}

Individuals start in a standing position and are allowed to use a countermovement, but they are not allowed to step into the jump. Individuals first have their max standing reach measured and then perform maximum jumps. Individuals hit the "flags" attached to the pole of the Vertex, which are spaced at half-inch increments. Jump height is recorded as distance between the max jump and the standing reach. 


\section{Appendix F: Southeast Missouri Configuration (SEMO) ${ }^{6}$}

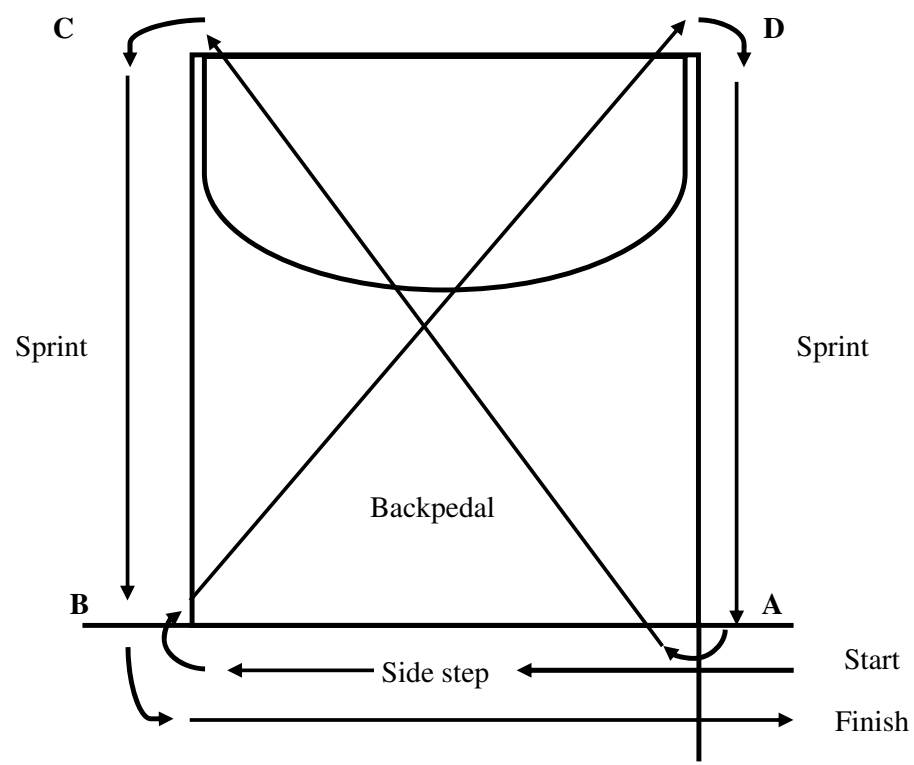

Individuals begin at the start with their back to the free-throw line. At "go" individuals will side step from A to B, then backpedal from $\mathrm{B}$ to $\mathrm{D}$, sprint forward from $\mathrm{D}$ to $\mathrm{A}$, again backpedal from $\mathrm{A}$ to $\mathrm{C}$, sprint forward from $\mathrm{C}$ to $\mathrm{B}$, and sidestep from $\mathrm{B}$ to the finish line at $\mathrm{A}$. Performance is measured by time elapsed to complete the task.

\section{Appendix G: Right-Boomerang Run Agility Test ${ }^{4}$}

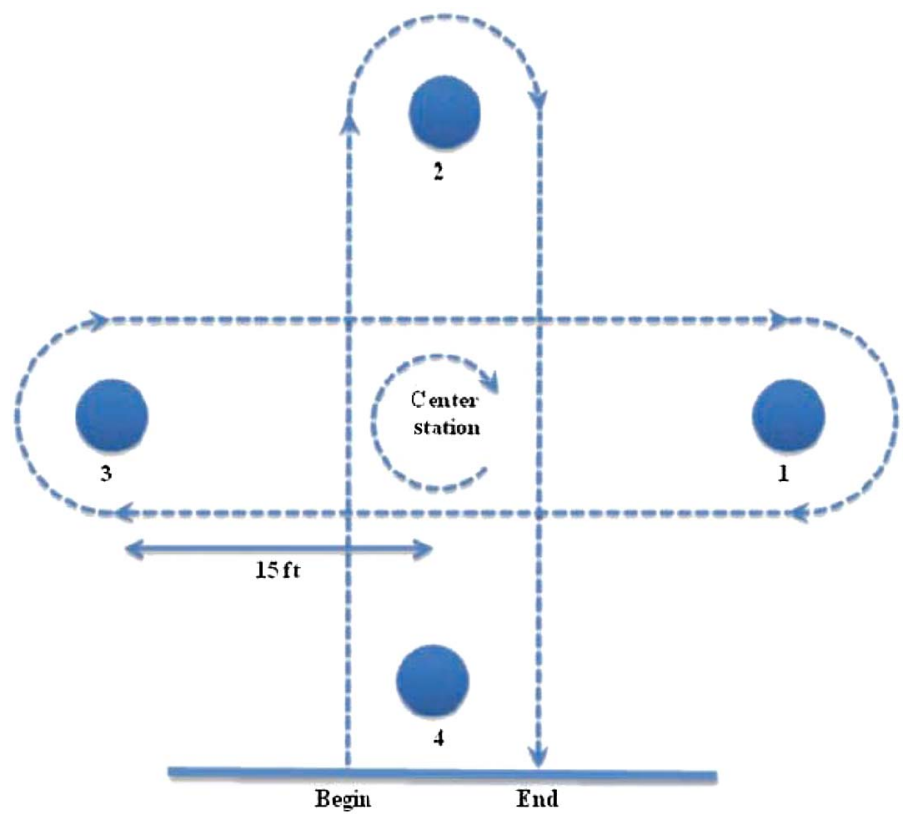

Cones are placed $15 \mathrm{ft}$ apart from the center station. Individuals on the signal "go" run to the center station, then make a quarter turn and run around the first outside station. Once the center station is reached, the individual again proceeds to make another quarter turn and run around the second outside station. This step is repeated for station 3, whereas for station 4 , the individual runs through the finish line instead of going back to the center circle. Performance is measured by time elapsed to complete the task. 


\section{Appendix H: $\mathbf{S} 180^{\circ}$ Run $^{3}$}

Individuals sprint $9 \mathrm{~m}$ from the start line to a point marked " 2 ." After touching the point with $1 \mathrm{ft}$, a $180^{\circ}$ left or right turn is made to go back to the starting line. Individuals then run $3 \mathrm{~m}$ to point " 3 " and make another $180^{\circ}$ turn, run $6 \mathrm{~m}$ forward to point " 4 " and make another $180^{\circ}$ turn. They will run $3 \mathrm{~m}$ to return to point " 2 " and then make 1 final turn to run $9 \mathrm{~m}$ to the finish line. Performance is measured by time elapsed to complete the task.

\section{Appendix I: $t$ Test $^{3}$}

The course is created using cones arranged in a " $\mathrm{T}$ " formation, $9.14 \mathrm{~m}$ in length and $9.14 \mathrm{~m}$ in width. Individuals sprint forward and touch the tip of the cone with their hand. They then will shuffle laterally to the left $4.57 \mathrm{~m}$ and touch the tip of the cone with their hand. Subsequently, they will proceed to shuffle across the distance of the "T" (9.14 m) to touch the right cone. Individuals will shuffle back to the center cone and touch it with their hand and then backpedal to the finish line (starting point). This is completed for time.

\section{Appendix J: Accuracy Shooting at a Target ${ }^{3}$}

A $1.22 \times 2.44-\mathrm{m}$ shooting target was constructed with a $5 \times 5-\mathrm{cm}$ "bull's-eye" in the center of the target. Each individual is instructed to kick a soccer ball from $6.1 \mathrm{~m}$ away from the base of the target and try to hit the "bull's-eye," as if shooting a goal. Measurements are taken from the center of the "bull's-eye" to the center of the ball mark to determine accuracy of shooting a soccer ball. 